Adriano Medeiros dos Santos

\title{
Suporte a Componentes Compostos Para o Middleware SCS
}

Dissertação apresentada ao Programa de Pós-graduação em Informática do Departamento de Informática do Centro Técnico Científico da PUC-Rio como requisito parcial para obtenção do grau de Mestre em Informática.

Orientador: Prof. Renato Fontoura de Gusmão Cerqueira 
Adriano Medeiros dos Santos

\title{
Suporte a Componentes Compostos Para o Middleware SCS
}

Dissertação apresentada ao Programa de Pós-graduação em Informática do Departamento de Informática do Centro Técnico Científico da PUC-Rio como requisito parcial para obtenção do grau de Mestre em Informática. Aprovada pela Comissão Examinadora abaixo assinada.

\author{
Prof. Renato Fontoura de Gusmão Cerqueira \\ Orientador \\ Departamento de Informática — PUC-Rio
}

Prof. Alessandro Fabricio Garcia Departamento de Informática — PUC-Rio

Prof. Antônio Tadeu Azevedo Gomes LNCC

Prof. José Eugenio Leal Coordenador Setorial do Centro Técnico Científico - PUC-Rio 
Todos os direitos reservados. É proibida a reprodução total ou parcial do trabalho sem autorização da universidade, do autor e do orientador.

\section{Adriano Medeiros dos Santos}

Graduou-se em Bacharelado em Ciência da Computação pela UESC. É pesquisador do laboratório Tecnologia em Computação Gráfica (Tecgraf) da PUC-Rio desde Agosto de 2009, onde trabalha no desenvolvimento do middleware SCS que apoia diversos projetos em parceria com a Petrobras S/A.

Ficha Catalográfica

Santos, Adriano Medeiros

Suporte a componentes compostos para o middleware scs / Adriano Medeiros dos Santos; orientador: Renato Fontoura de Gusmão Cerqueira. - Rio de Janeiro : PUC-Rio, Departamento de Informática, 2012.

v., 99 f: il. ; $29,7 \mathrm{~cm}$

1. Dissertação (mestrado) - Pontifícia Universidade Católica do Rio de Janeiro, Departamento de Informática.

Inclui referências bibliográficas.

1. Informática - Tese. 2. Modelos de Componentes de Software. 3. Programação Orientada a Componentes. 4. Componentes Compostos. 5. Middleware. I. Cerqueira, Renato. II. Pontifícia Universidade Católica do Rio de Janeiro. Departamento de Informática. III. Título. 


\section{Agradecimentos}

Ao meu pai Josembergue Campos dos Santos, meu exemplo de como ser uma pessoa dedicada ao trabalho e sempre me ajudar em decisões como por exemplo investir neste mestrado.

À minha mãe Joíse Cristina Medeiros dos Santos, pelo carinho e amor que me deram força para este trabalho.

À minha namorada Tiara, pelo amor e compreensão da necessidade do namoro à distância.

Ao meu orientador Renato Cerqueira, que acreditou em meu trabalho e me inspirou a alcançar meus objetivos.

À PUC-Rio e Tecgraf, pelos infraestrutura, sem os quais este trabalho não poderia ter sido realizado.

Ao Amadeu pelas explicações e por me mostrar que sempre é bom pensar bem antes de discutir uma idéia.

Aos meus amigos, que fizeram com que esse mestrado no Rio de Janeiro longe da família fosse bem animado. 


\section{Resumo}

Santos, Adriano Medeiros; Cerqueira, Renato. Suporte a Componentes Compostos Para o Middleware SCS. Rio de Janeiro, 2012. 99p. Dissertação de Mestrado — Departamento de Informática, Pontifícia Universidade Católica do Rio de Janeiro.

Diversos modelos de componentes de software apresentam o conceito de componentes compostos, como é o caso do Fractal, OpenCOM, Koala, Kobra e SaveCCM. Um componente composto encapsula outros componentes e é responsável por oferecer mecanismos de configuração e introspecção estrutural, bem como externalizar serviços e dependências internas. A idéia de componentes compostos é reconhecida como uma boa técnica para abstrair estruturas complexas e maximizar o reuso. Entretanto, não existe um estudo mais aprofundado sobre tal mecanismo que aborde questões sobre a configuração inicial dos subcomponentes, o mapeamento de serviços e dependências de subcomponentes através de interfaces do componente composto, formas de navegação, compartilhamento de subcomponentes, ciclo de vida e formas de introspecção estrutural. Neste trabalho foi realizado um levantamento sobre como os modelos Fractal e OpenCOM tratam o conceito de componentes compostos e é proposto um modelo que oferece este suporte. Como estudo experimental foi implementada uma nova versão do SCS com suporte a componentes compostos, o SCS-Composite. Também foi feita uma avaliação da expressividade do SCS-Composite através da adaptação de uma aplicação pré-existente baseada no SCS.

\section{Palavras-chave}

Modelos de Componentes de Software. Programação Orientada a Componentes. Componentes Compostos. Middleware. 


\section{Abstract}

Santos, Adriano Medeiros; Cerqueira, Renato (Advisor). Composite Component Support for the SCS Middleware. Rio de Janeiro, 2012. 99p. MSc Dissertation — Departamento de Informática, Pontifícia Universidade Católica do Rio de Janeiro.

Several software component models support composite components, such as Fractal, OpenCOM, Koala, Kobra and SaveCCM. A composite component encapsulates components and provides introspection and configuration mechanisms. Composite components are recognized as a good practice because abstract complex structures and increase reuse. However, there is not a comprehensive study about composite component mechanisms addressing issues like initial composite configuration, mapping of subcomponents' services and dependencies through composite component's interfaces, introspection, component sharing, and composite's life cycle. In this work we perform a study about how the Fractal and OpenCOM apply the composite component concept and we propose a new model for composite components. As an experimental study we implemented a new version of the SCS component system with support for composite components, the SCS-Composite. We also present an evaluation of SCS-Composite through the adaptation of a SCS based application.

\section{Keywords}

Software Component Models. Component-oriented programming. Composite Component. Middleware. 


\section{Sumário}

1 Introdução $\quad 12$

$\begin{array}{lll}1.1 & \text { Objetivos e Contribuições } & 15\end{array}$

$\begin{array}{lll}1.2 & \text { Estrutura do Documento } & 16\end{array}$

2 Trabalhos Relacionados $\quad 18$

2.1 Fractal 19

2.2 OpenCOM 22

2.3 Características de Componentes Compostos 26

2.3.1 Suporte ao Desenvolvimento Incremental 26

2.3.2 Mapeamento de Serviços dos Subcomponentes como Serviços do Componente Composto 27

2.3.3 Mapeamento de dependências de subcomponentes como dependências do componente composto $\quad 29$

2.3.4 Conectores para Binding Vertical $\quad 31$

2.3.5 Relacionamento Serviço/Componente 32

2.3.6 Compartilhamento de subcomponentes 34

2.3.7 Navegação entre Subcomponente e Componente Composto 36

2.3.8 Mecanismos de Reconfiguração 36

2.4 Considerações Finais 37

3 Modelo SCS com Suporte a Componentes Compostos 39

3.1 Modelo de Componentes SCS 39

$\begin{array}{lll}3.1 .1 & \text { Componente } & 39\end{array}$

3.1.2 Mecanismo de Binding 40

3.2 Modelo de Componentes SCS-Composite 43

3.2.1 Componente Composto 43

3.2.2 Hierarquia de Componentes 44

3.2.3 Externalização de Facetas e Receptáculos 44

3.2.4 Regras de Conexão 46

3.3 Diretrizes do Modelo 47

3.3.1 Suporte ao Desenvolvimento Incremental 47

3.3.2 Mapeamento de Serviços dos Subcomponentes como serviços do componente composto e Conectores Exógenos 48

3.3.3 Relacionamentos Serviço/Componente e Subcomponente/Componente Composto 50

3.3.4 Mapeamento de Dependências de Subcomponentes como Dependências do Componente Composto 50

3.3.5 Compartilhamento de subcomponentes $\quad 51$

3.3.6 Mecanismos de Reconfiguração $\quad 52$

3.4 Considerações Finais $\quad 52$

4 Middleware SCS-Composite $\quad 55$

4.1 Middleware SCS $\quad 55$

4.2 Middleware SCS-Composite $\quad 57$ 
$\begin{array}{lll}\text { 4.2.1 Estrutura do Componente } & 57\end{array}$

4.2.2 Mecanismo de binding 60

4.3 Exemplo de Uso 62

4.3.1 Mapeamento de Facetas $\quad 62$

4.3.2 Mapeamento de Receptáculos 63

$\begin{array}{lll}4.4 & \text { Considerações Finais } & 65\end{array}$

$\begin{array}{lll}5 & \text { Exemplo de Uso } & 67\end{array}$

$\begin{array}{lll}5.1 & \text { Modelagem do CAS } & 67\end{array}$

5.2 Implementação com Middleware SCS 68

5.3 Implementação com Middleware SCS-Composite 71

5.3.1 Gerência sobre Componentes $\quad 71$

5.3.2 Mapeamento de Serviços Internos $\quad 72$

$\begin{array}{lll}\text { 5.3.3 Binding Vertical com Aridade 1-n } & 72\end{array}$

5.3.4 Gerência de Configuração da Aplicação 73

5.4 Comparação do CAS: SCS Vs. SCS-Composite 74

$\begin{array}{lll}6 & \text { Conclusão } & 77\end{array}$

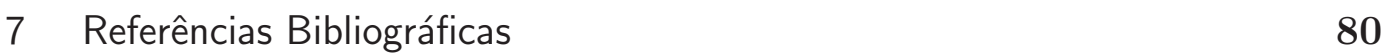

A Middleware SCS $2.0 \quad 83$

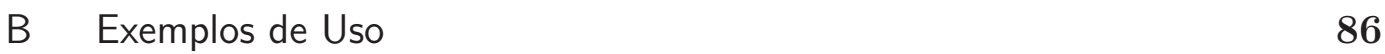

B.1 Mapeamento de Facetas $\quad 86$

B.2 Mapeamento de Receptáculos 90

$\begin{array}{lll}\text { C Interfaces do CAS } & 96\end{array}$ 


\section{Lista de figuras}

1.1 Sistema baseado em componentes. Em 1 é ilustrado o binding entre dois componentes; e em 2, é ilustrado binding entre um componente e a plataforma operacional. 13

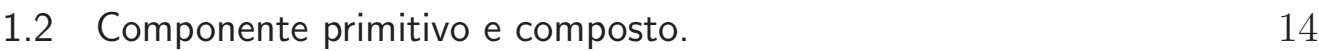

1.3 Representação dos dois tipos de bindings verticais 14

2.1 Estrutura de um componente no modelo Fractal. 19

2.2 Arquitetura do modelo de componentes OpenCOM (Michael Clarke, 2007). 23

2.3 Um Component Framework em OpenCOM. 24

2.4 Mapeamentos de serviços dos subcomponentes através de interfaces do componente composto. 27

2.5 Exemplo de um cenário do CAS demonstrando a necessidade de disponibilizar serviços internos por interfaces externas diferentes. 28

2.6 Mapeamentos de dependências dos subcomponentes através de interfaces externas do componente composto.

2.7 Arquitetura de uma interface gráfica com compartilhamento de subcomponentes.

2.8 Cenário onde o compartilhamento de subcomponentes nos leva a uma situação de ambiguidade quanto as dependências do componente compartilhado.

3.1 Representação de um componente SCS.

3.2 Exemplo de conexão entre facetas e receptáculos de componentes SCS.

3.3 Exemplo de uma composição formada pelos componentes A, B e C. 42

3.4 Os dois tipos de receptáculos presentes no SCS-Composite 46

3.5 Exemplos de conexões entre componentes primitivos e compostos. 48

3.6 Mapeamento de uma faceta de um subcomponente para facetas externas diferentes.

3.7 Funcionamento de um conector no processo de externalização de facetas.

3.8 Representação de externalização de um receptáculo de um subcomponente.

4.1 Os dois tipos de receptáculos presentes no SCS-Composite

4.2 Arquitetura do exemplo de uso do SCS-Composite Local

4.3 Arquitetura do exemplo de uso do SCS-Composite Distribuído.

5.1 Modelagem realizada para arquitetura atual do CAS.

5.2 Implementação do CAS com o SCS sem suporte a componentes compostos.

5.3 Exemplo do uso de conectores no CAS. 


\section{Lista de tabelas}

2.1 Comparação entre os modelos Fractal e OpenCOM sobre características de componentes compostos em conjunto com as decisões realizadas para o modelo SCS. 
A mente que se abre a uma nova idéia jamais voltará ao seu tamanho original."

Albert Einstein (1879-1955) 